\title{
Non-reductive Homolytic Scission of Endoperoxide Bond for Activation of Artemisinin: The Bi-radical Perspectives
}

\author{
Shikha Sharma ${ }^{1}$ and Ehesan Ali $^{1}$ \\ ${ }^{1}$ Institute of Nano Science and Technology
}

July 6, 2021

\begin{abstract}
Artemisinin is the most successful antimalarial drug against malaria caused by Plasmodium falciparum. Despite its tremendous success and popularity in malaria therapeutics, the molecular mechanism of artemisinin's activity is still elusive. The activation of artemisinin, i.e., cleavage of the endoperoxide bond at the infected cell that generates radical intermediates and the subsequent chemical rearrangements plays a key role in the antimalarial activities. In this work, applying state-of-the-art computational techniques based on the spin-constraint density functional theory (CDFT) along with ab initio thermodynamics, we have investigated various key steps of the molecular mechanism of artemisinin. The well-accepted artemisinin activation process is the reductive heterolytic scission of the endo-peroxide bond which is followed by subsequent chemical reactions that propagate via mono-radical intermediates. Adopting CDFT methodology, here we have investigated the possible alternative 'biradical' intermediates and their mechanistic pathways for the subsequent chemical reactions. The change in Gibbs free energy associated with the activation of artemisinin through homolytic-scissoring (biradical) intermediate is quite competitive and favorable compared to the reductive heterolytic-scissoring (monoradical) process. This indicates the alternative possibilities for the biradical activation process. The reported experimental EPR signals for the biradicals especially for similar anti-malarial drugs like G3-factor support our observations.
\end{abstract}

\section{Hosted file}

Art_manuscript.docx available at https://authorea.com/users/424070/articles/529233-nonreductive-homolytic-scission-of-endoperoxide-bond-for-activation-of-artemisinin-the-biradical-perspectives 